\title{
ARTICLE
}

Molecular Diagnostics

\section{Poor outcome in hypoxic endometrial carcinoma is related}

\section{to vascular density}

Casper Reijnen ${ }^{1,2}$, Willem Jan van Weelden ${ }^{1}$, Martijn S. J. P. Arts ${ }^{3}$, Johan P. Peters ${ }^{3}$, Paul F. Rijken ${ }^{3}$, Koen van de Vijver ${ }^{4}$, Maria Santacana ${ }^{5}$, Peter Bronsert ${ }^{6}$, Johan Bulten ${ }^{7}$, Marc Hirschfeld ${ }^{8,9}$, Eva Colas ${ }^{10}$, Antonio Gil-Moreno ${ }^{10,11}$, Armando Reques ${ }^{12}$, Gemma Mancebo ${ }^{13}$, Camilla Krakstad ${ }^{14,15}$, Jone Trovik ${ }^{14}$, Ingfrid S. Haldorsen ${ }^{15,16}$, Jutta Huvila ${ }^{17}$, Martin Koskas ${ }^{18}$, Vit Weinberger ${ }^{19}$, Lubos Minar ${ }^{19}$, Eva Jandakova ${ }^{20}$, Marc P. L. M. Snijders ${ }^{2}$, Saskia van den Berg-van Erp ${ }^{21}$, Heidi V. N. Küsters-Vandevelde ${ }^{21}$, Xavier Matias-Guiu ${ }^{5}$, Frederic Amant ${ }^{22,23}$, ENITEC-consortium, Leon F. A. G. Massuger ${ }^{1}$, Johan Bussink ${ }^{3}$ and Johanna M. A. Pijnenborg ${ }^{1}$

BACKGROUND: Identification of endometrial carcinoma (EC) patients at high risk of recurrence is lacking. In this study, the prognostic role of hypoxia and angiogenesis was investigated in EC patients.

METHODS: Tumour slides from EC patients were stained by immunofluorescence for carbonic anhydrase IX (CAIX) as hypoxic marker and CD34 for assessment of microvessel density (MVD). CAIX expression was determined in epithelial tumour cells, with a cut-off of $1 \%$. MVD was assessed according to the Weidner method. Correlations with disease-specific survival (DSS), disease-free survival (DFS) and distant disease-free survival (DDFS) were calculated using Kaplan-Meier curves and Cox regression analysis. RESULTS: Sixty-three (16.4\%) of 385 ECs showed positive CAIX expression with high vascular density. These ECs had a reduced DSS compared to tumours with either hypoxia or high vascular density (log-rank $p=0.002)$. Multivariable analysis showed that hypoxic tumours with high vascular density had a reduced DSS (hazard ratio [HR] 3.71, $p=0.002$ ), DDFS (HR 2.68, $p=0.009$ ) and a trend for reduced DFS (HR 1.87, $p=0.054)$.

CONCLUSIONS: This study has shown that adverse outcome in hypoxic ECs is seen in the presence of high vascular density, suggesting an important role of angiogenesis in the metastatic process of hypoxic EC. Differential adjuvant treatment might be indicated for these patients.

British Journal of Cancer (2019) 120:1037-1044; https://doi.org/10.1038/s41416-019-0461-2

\section{BACKGROUND}

Most endometrial carcinoma (EC) patients present with early-stage disease and have a favourable outcome. Nevertheless, $15 \%$ of all patients suffer from recurrent disease and subsequently have a poor outcome. ${ }^{1-3}$ Approximately half of these recurrences occur in patients primarily diagnosed with low-risk EC. ${ }^{1,4}$ Improved identification of patients at high risk for recurrence is crucial to prevent both over- and undertreatment.

Hypoxia is known to be an important feature of aggressive EC and drives metastatic potential. ${ }^{5-8}$ When solid tumours outgrow their vasculature beyond the size of $0.1 \mathrm{~mm}^{3}$, hypoxia may occur. ${ }^{9}$ As a response to chronic hypoxia, tumour cells will activate genes associated with more aggressive phenotype and resistance to chemotherapy and radiotherapy. ${ }^{10}$ Hypoxia-inducible factor 1 (HIF-1), formed after heterodimerisation of its subunits HIF-1a and HIF-1 $\beta$, plays a key role in this process. ${ }^{11,12}$ HIF-1 activates downstream genes that enhance cell survival by maintaining intracellular $\mathrm{pH}$, stimulating angiogenesis to increase oxygen delivery and switching to anaerobic glycolysis. ${ }^{12,13}$ More specifically, an important downstream target is carbonic anhydrase 9

\footnotetext{
${ }^{1}$ Department of Obstetrics and Gynaecology, Radboud Institute for Health Sciences, Radboud University Medical Center, Nijmegen, The Netherlands; ${ }^{2}$ Department of Obstetrics and Gynaecology, Canisius-Wilhelmina Hospital, Nijmegen, The Netherlands; ${ }^{3}$ Department of Radiation Oncology, Radboud University Medical Center, Nijmegen, The Netherlands; ${ }^{4}$ Department of Pathology, Ghent University Hospital, Cancer Research Institute Ghent (CRIG), Ghent, Belgium; ${ }^{5}$ Department of Pathology and Molecular Genetics and Research Laboratory, Hospital Universitari Arnau de Vilanova, University of Lleida, IRBLleida, CIBERONC, Lleida, Spain; ${ }^{6}$ Institute of Pathology, University Medical Center, Freiburg, Germany; ${ }^{7}$ Department of Pathology, Radboud University Medical Center, Nijmegen, The Netherlands; ${ }^{8}$ Department of Obstetrics and Gynecology, University Medical Center, Freiburg, Germany; ${ }^{9}$ German Cancer Consortium, German Cancer Research Center, Heidelberg, Germany; ${ }^{10}$ Biomedical Research Group in Gynecology, Vall Hebron

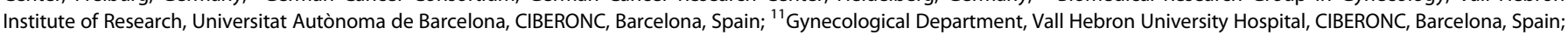
${ }^{12}$ Pathology Department, Vall Hebron University Hospital, CIBERONC, Barcelona, Spain; ${ }^{13}$ Department of Obstetrics and Gynecology, Hospital del Mar, PSMARB, Barcelona, Spain; ${ }^{14}$ Department of Obstetrics and Gynecology, Haukeland University Hospital, Bergen, Norway; ${ }^{15}$ Centre for Cancer Biomarkers, Department of Clinical Science, University of Bergen, Bergen, Norway; ${ }^{16}$ Mohn Medical Imaging and Visualization Centre, Department of Radiology, Haukeland University Hospital, Bergen, Norway; ${ }^{17}$ Department of Pathology, University of Turku, Turku, Finland; ${ }^{18}$ Obstetrics and Gynecology Department, Bichat-Claude Bernard Hospital, Paris, France; ${ }^{19}$ Department of Gynecology and Obstetrics, Faculty of Medicine, Masaryk University, Brno, Czech Republic; ${ }^{20}$ Institute of Pathology, Faculty of Medicine, Masaryk University, Brno, Czech Republic; ${ }^{21}$ Department of

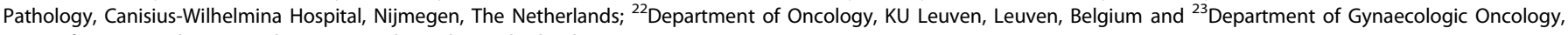
Center for Gynaecologic Oncology, Amsterdam, The Netherlands
}

Correspondence: Casper Reijnen (Casper.reijnen@radboudumc.nl)

These authors contributed equally: Casper Reijnen, Willem Jan van Weelden.

Received: 5 December 2018 Revised: 1 April 2019 Accepted: 4 April 2019

Published online: 23 April 2019 
(CA9), whose encoded protein, carbonic anhydrase IX (CAIX), regulates intracellular $\mathrm{pH}$ by converting carbon dioxide to carbonic acid. ${ }^{14}$ By adaptation of tumour cells to a hostile microenvironment, tumour proliferation can commence even in hypoxic areas. ${ }^{15}$ Also in normoxic conditions, HIF-1 can be activated; however, downstream activation is present in lesser extent. ${ }^{16,17}$ In this perspective CAIX expression, one of the key effector proteins of HIF-1, has been shown to be more specifically related to hypoxia and poor outcome. ${ }^{18}$

Next to maintenance of intracellular $\mathrm{pH}$, stimulation of angiogenesis is an important response to hypoxia. Vascular endothelial growth factor (VEGF), another downstream target of HIF-1, is also correlated with hypoxia and angiogenesis in several cancer types, including EC. ${ }^{19-22}$ Angiogenesis can be assessed by microvessel density (MVD) and is prognostically associated with deep myometrial invasion (MI), lymphovascular space invasion (LVSI) and poor overall survival in EC. ${ }^{23}$ Although earlier studies suggest correlation between hypoxia, angiogenesis and poor outcome, the prognostic value has not yet been studied before. ${ }^{5,6}$ Therefore, we have investigated the prognostic value of hypoxia and angiogenesis in EC, assessed with CAIX expression and MVD.

\section{METHODS}

\section{Patients}

Data and tumour slides were collected previously for a study analysing the value of L1CAM expression in ECs, which included ECs from 11 collaborating European Network for Individualised Treatment of Endometrial Cancer (ENITEC) centres. ${ }^{24,25}$ Only cases diagnosed by an expert gynaecological pathologist, with complete data on treatment and pathological examination and at least 36 months of follow-up, were included. Cases with a nonendometrioid component were categorised as non-endometrioid. The 1199 cases included in the original study were randomly selected using SPSS version 22 (SPSS IBM, New York, NY, USA), resulting in a database of 403 patients for the present study. These cases were not statistically different from the original cases for the most important baseline characteristics.

\section{Tissue and staining}

Four micrometre sections, derived from formalin-fixed, paraffinembedded ECs were used to visualise CAIX and blood vessels. Sections were mounted on Superfrost slides (Menzel-Gläser). Slides were deparaffinated in Histochoise (VWR H103-4L) and rehydrated (graded ethanol: $100-96-70 \%$ and de-ionised water). Next, citrate buffer antigen retrieval was performed for $30 \mathrm{~min}$ (Target retrieval solution $10 \times, \mathrm{pH} 6$ citrate, Dako Cytomation, $96^{\circ} \mathrm{C}$ ). Prior to incubation with the primary antibodies, sections were blocked with 5\% normal goat serum (Jackson ImmunoResearch) in primary antibody diluent (PAD, BIORAD BUF014), 30 min at room temperature. Thereafter, sections were co-stained for CAIX (Novus Biologicals NB100-417, 1:100) and vessels (CD34, ABCAM ab8536, 1:300), $60 \mathrm{~min}$ at $37^{\circ} \mathrm{C}$. Secondary incubation was performed using $\mathrm{Cy}^{\mathrm{TM}_{3}}$ Fab Fragment Goat Anti-Rabbit immunoglobulin $\mathrm{G}(\mathrm{IgG})(\mathrm{H}+\mathrm{L}$ ) polyclonal IgG (Jackson ImmunoResearch 111-167-003) for CAIX and CF ${ }^{\oplus} 488$ a Goat anti-Mouse lgG $(\mathrm{H}$ $+\mathrm{L}), \mathrm{F}\left(\mathrm{ab} \mathrm{b}^{\prime}\right) 2$ fragment polyclonal lgG (Biotium CF488A) for the vessels, $60 \mathrm{~min}$ at $37^{\circ} \mathrm{C}$. All antibodies were diluted in PAD. In between stainings, sections were rinsed with phosphate-buffered saline (JT Baker 4391.9010). 4',6-Diamidino-2-phenylindole (DAPI) (Santa Cruz Biotechnology AB-17.0097) was used as a counterstain to stain all nuclei, and finally the sections were mounted with Fluoromount W (Serva 21634.01). Haematoxylin and eosin staining was used for morphological evaluation. CAIX expression was scored as the fraction of epithelial tumour cells with positive membranous staining.
Image analysis

Tumour slides were analysed using a digital image analysis system after scanning of the whole slides with the Axio Imager D2 microscope (Carl Zeiss, GmbH, Oberkochen, Germany) using a Prior lumen 200 metal halide lamp (Prior Scientific, Rockland, USA), Axiocam 503 mono 16-bit camera $(1936 \times 1460$ pixel, Carl Zeiss, $\mathrm{GmbH}$ ) and a computer-controlled motorised stage (Carl Zeiss, $\mathrm{GmbH}$ ) directed by Zen Pro software (Carl Zeiss, GmbH). ${ }^{26}$ Each slide was scanned for three signals: DAPI (all nuclei), Alexa488 (CD34) and Cy3 (CAIX), by means of a 10x objective using standardised shutter times for each signal $(1,25$ and $50 \mathrm{~ms}$, respectively). After scanning, grey-scale images of all three recorded signals were used for analysis.

For analysis of CAIX staining, only membranous expression on epithelial tumour cells was analysed. Areas of necrosis, large vessels and tumour stroma, determined using H\&E-stained adjacent tumour slides, were therefore manually excluded from the analysis (i-Vision for Mac; BioVision Technologies, Exton, PA, USA). Next, thresholds for segmentation of the fluorescent signals were interactively set above the background staining for each individual marker and adjusted for each sample in order to optimise the signal to background ratio using ImageJ software (Wayne Rasband, National Institute of Mental Health, National Institutes of Health). An interactively set threshold limits intersample variability by correction for differences in immunofluorescence staining intensity. ${ }^{26,27}$ The resulting binary images were used to calculate the fraction of CAIX (fCAIX) relative to the total tumour area. To minimise bias of non-specific staining, only positive signals exceeding 5 pixels were included.

The MVD was measured according to the Weidner method. ${ }^{28}$ In short, surrounding epithelial tumour cells three areas with the highest density of vessels were selected by the assessor (M.A.) using a $\times 200$ magnification. To correct for objects that exceed the image borders, only objects exceeding the left and upper border were included. To minimise bias of nonspecific staining, only positive signals exceeding 2 pixels were included.

CAIX expression was considered positive when the fCAIX was above $1 \% .^{29,30}$ The MVD was dichotomised over the median. A representative example of CAIX and MVD staining is shown in Fig. 1.

\section{Statistical analyses}

Clinicopathological differences between subgroups were compared with the $X^{2}$ and Fisher's exact tests for categorical data and the Mann-Whitney $U$ test for continuous variables.

Kaplan-Meier curves were constructed for disease-specific survival (DSS), disease-free survival (DFS) and distant-DFS (DDFS). The association between CAIX and MVD and DSS, DFS and DDFS was determined using Cox regression analysis. DSS was calculated from the date of primary treatment to the date of death caused by the disease or, for surviving patients, to the date of the last followup. DFS and DDFS were defined as the length of follow-up, after completion of the primary treatment, during which women survived without any clinical sign of (distant) disease recurrence. Distant recurrence included metastases in distant organs and para-aortic lymph nodes. Features identified by univariable regression analysis with $p<0.20$ were used for multivariable regression analysis. LVSI was coded as negative in case of missing data $(n=108)$ since only substantial LVSI was recently reported as relevant for prognosis of EC. If LVSI was not reported in the pathological report, it was therefore assumed that LVSI was absent. $^{31,32} P$ values $<0.05$ were considered to indicate a significant difference. SPSS version 25 (SPSS IBM, New York, NY, USA) statistical software was used to perform the statistical analyses. 

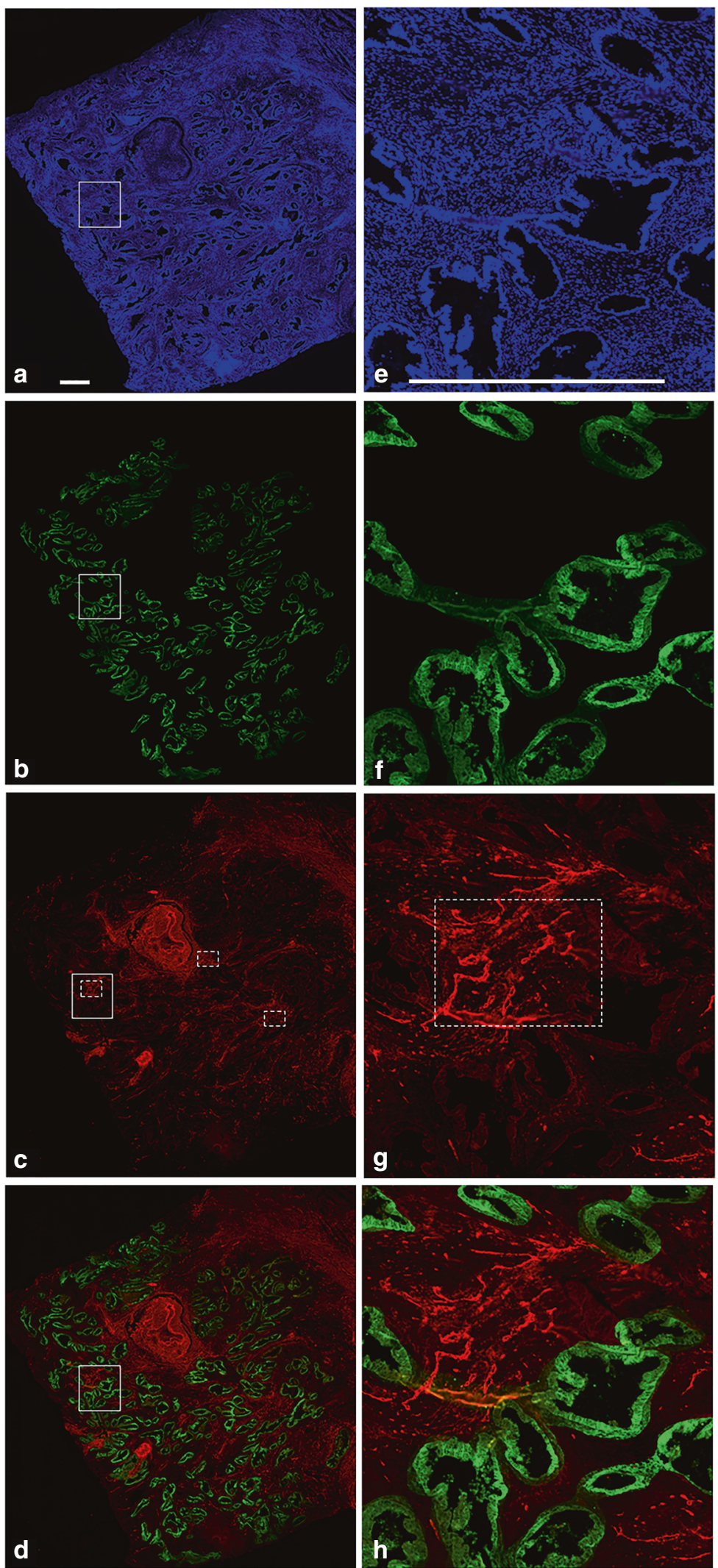

Fig. 1 Example of carbonic anhydrase IX (CAIX) and CD34 staining in endometrial cancer. a Nuclear 4',6-diamidino-2-phenylindole (DAPI) staining (blue) for visualisation of tumour nuclei. b CAIX staining (green) adjusted for total tumour area, meaning that only epithelial tumour cells were included in the analysis: other tissue, including stroma, necrosis and vasculature has manually been removed and is coloured black by the analysis software (see "Methods" section). c CD34 staining of vasculature (red) with three hotspots according to the Weidner method, marked with the interrupted lines. $\mathbf{d}$ Combined CAIX staining and CD34 staining. $\mathbf{e}-\mathbf{h}$ represent representative high-magnification images of the boxed areas in $\mathbf{a}, \mathbf{b}, \mathbf{c}$ and $\mathbf{d}$, respectively. Scale bar $=0.5 \mathrm{~mm}$ 


\section{RESULTS}

Patients

After staining for CAIX and CD34, 18 of the 403 patients were excluded due to insufficient tumour tissue $(n=9)$ and excess of nonspecific background staining $(n=9)$. Clinicopathological characteristics of the 385 patients included for analysis are shown in Table 1. Overall, the median age was 64 years and the median follow-up time was 58 months. Of all patients alive at the end of follow-up, $99 \%$ had a follow-up of at least 36 months. A total of 67 patients (17.4\%) were diagnosed with high-grade EC, including 13 non-endometrioid endometrial carcinomas (NEECs) (3.4\%). In total, 106 (27\%) EC patients had positive CAIX expression. Forty-seven patients $(12.2 \%)$ recurred and 21 patients (5.5\%) died due to the disease. Of all the patients with recurrence, 14 (3.6\%) had a local recurrence, $16(4.2 \%)$ a regional recurrence and 31 (8.1\%) a distant recurrence.

\section{CAIX expression and MVD}

A total of 63 carcinomas (16.4\%) showed a positive membranous epithelial CAIX expression and high degrees of vascular density, defined as a MVD above the median (Table 1). CAIX expression with high vascular density was correlated with non-endometrioid histology $(7.9 \%$ vs. $2.5 \%, p=0.028)$, but not with other clinicopathological features. Patients with CAIX-positive ECs and high vascular density experienced more recurrences $(22.2 \%$ vs. $10.2 \%, p=0.008)$ and specifically more distant recurrences $(19.0 \%$ vs. $5.9 \%, p<0.001)$, as well as higher overall mortality $(22.2 \%$ vs. $11.8 \%, p=0.027)$ and EC-related mortality $(17.5 \%$ vs. $3.7 \%, p<$ 0.001) (Table 1).

Figure 2 shows that CAIX expression with high vascular density was associated with a worse DSS compared to CAIX expression with low vascular density and negative CAIX expression $(p=$

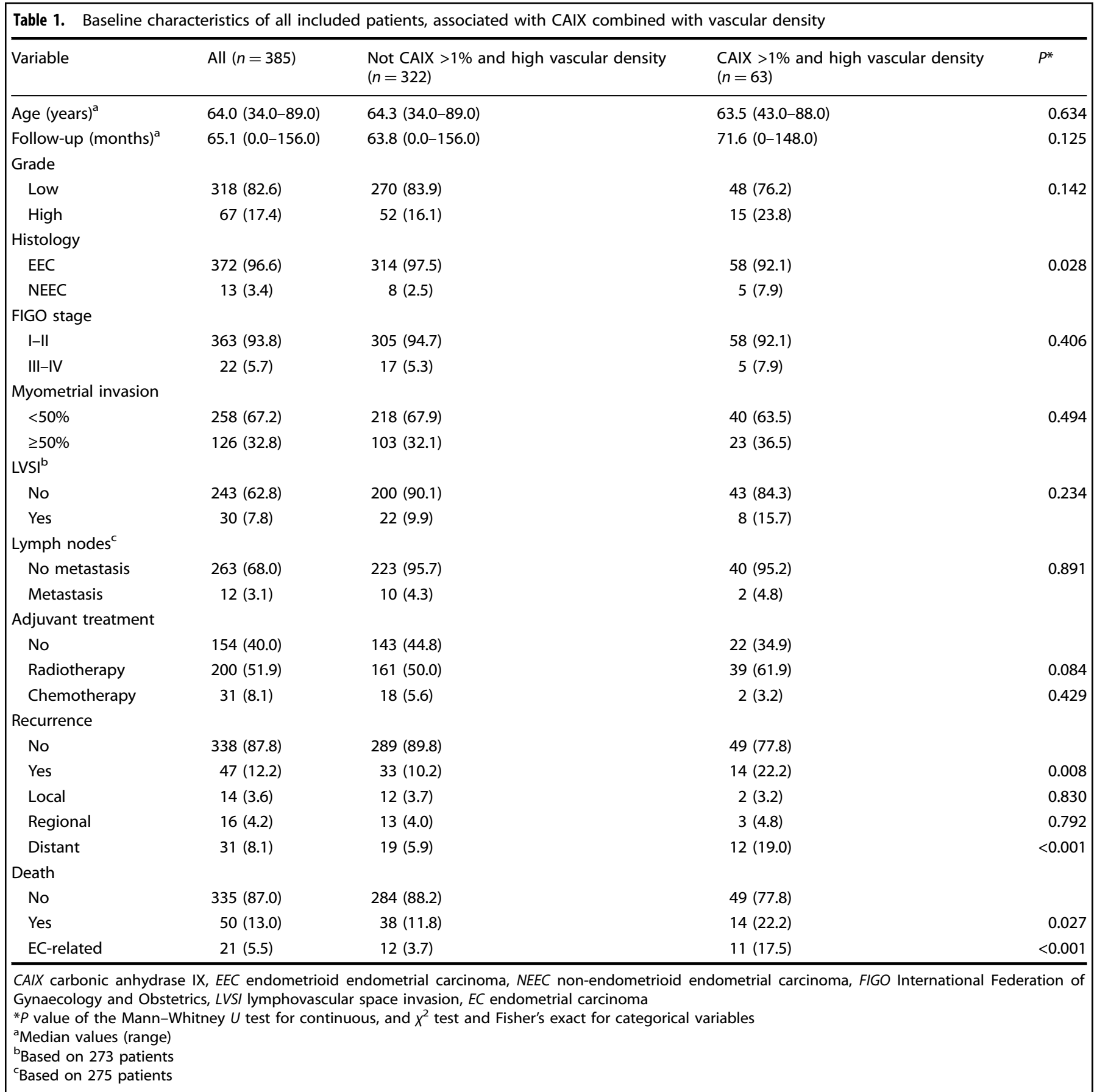


0.002). Interestingly, CAIX-positive ECs with low vascular density had a similar outcome as CAIX-negative ECs. Univariable Cox regression analysis revealed that age, CAIX expression with high vascular density, myocardial infarction (MI), International Federation of Gynaecology and Obstetrics (FIGO) stage, grade and lymphovascular space invasion (LVSI) were significantly associated with DSS (Fig. 3). In multivariable analysis, high age, CAIX expression with high vascular density and tumour grade 3 remained significantly associated with reduced DSS, with CAIX and MVD as the most significant parameter (hazard ratio [HR] 3.71, $95 \%$ confidence interval $(\mathrm{Cl}) 1.59-8.63, p=0.002)$.

Multivariable analysis showed that age, FIGO stage and LVSI were significantly associated with DFS. CAIX expression with high vascular density was nearly significant (HR 1.87, 95\% Cl 0.99-3.55, $p=0.054$, Fig. 4). Multivariable analysis for DDFS showed that LVSI and CAIX expression with high vascular density was significantly associated with a reduced DDFS (CAIX and MVD: HR $2.68,95 \% \mathrm{CI}$ 1.27-5.65, $p=0.009$, Fig. 5).

Individual contribution of CAIX and MVD

Positive CAIX expression was associated with high tumour grade, non-endometrioid histology, higher median MVD and treatment with radiotherapy. In multivariable analysis, CAIX and grade were significantly associated with DSS (HR 2.45, 95\% Cl 1.05-5.73, $p=$ 0.039) (Supplementary Tables 1 and 2). High MVD was correlated

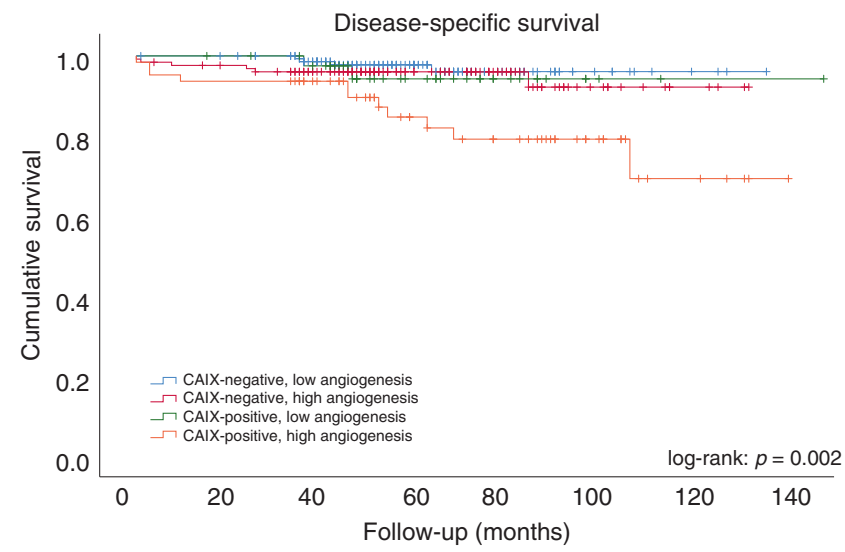

Fig. 2 Disease-specific survival (DSS) by carbonic anhydrase IX (CAIX) expression combined with degree of angiogenesis. Log-rank test was used to compare groups with deep Ml, but not with other clinicopathological factors. In multivariable analysis, high MVD remained an independent predictor of reduced DSS (HR 2.92, 95\% Cl 1.13-7.54, $p=0.027$ ) (Supplementary Tables 3 and 4). Continuous scoring of CAIX expression showed a significant correlation with DSS as well (data not shown).

\section{DISCUSSION}

In the present study, we have investigated the prognostic value of angiogenesis and hypoxia, assessed with MVD and CAIX expression. We hypothesised that angiogenesis would facilitate haematogenous spread of hypoxic tumour cells with subsequent poor clinical outcome. Additionally, we assumed that this would specifically be facilitated in hypoxic ECs, because of activation of intracellular pathways that induce an aggressive and metastatic phenotype. We have shown that CAIX expression with high vascular density is associated with reduced DSS and DDFS. Interestingly, CAIX-positive ECs with low vascular density had a similar outcome as CAIX-negative ECs. Finally, multivariable analyses for CAIX expression and vascular density showed that both were independent prognostic markers as well.

This is the largest study to date studying CAIX in EC. In contrast to previous studies in EC, we did find significant correlations between CAIX expression and poor outcome, especially in case of high vascular density. Seeber et al. ${ }^{29}$ included 93 patients and found CAIX expression in $76 \%$ of $\mathrm{ECs}^{29}$ In this study, no correlation between CAIX expression and outcome was found; however, small sample size and different cut-off value (all degrees of positive staining were regarded as positive) could explain why no correlation was found. Similarly, Pijnenborg et al. ${ }^{22}$ investigated CAIX expression in $59 \mathrm{ECs}$ and did not find a correlation. Again, possibly this study was underpowered due to a limited sample size and low number of distant recurrences. Also, differences in study design (case-control study) hamper valid comparison with our results. In other cancer types, including breast carcinoma, hepatocellular carcinoma, cervical carcinoma and renal cell carcinoma, CAIX expression is associated with poor prognosis. ${ }^{33-37}$ More specifically, increased distant failure was seen in several solid tumour types with positive CAIX expression. ${ }^{37,38}$

The metastatic process is a complex step-wise process, including acquisition of an aggressive phenotype, invasion in surrounding tissues and blood vessels, survival in the circulation with subsequent extravasation and colonisation in new organs. ${ }^{39}$ Hypoxia and subsequent neoangiogenesis will intervene with

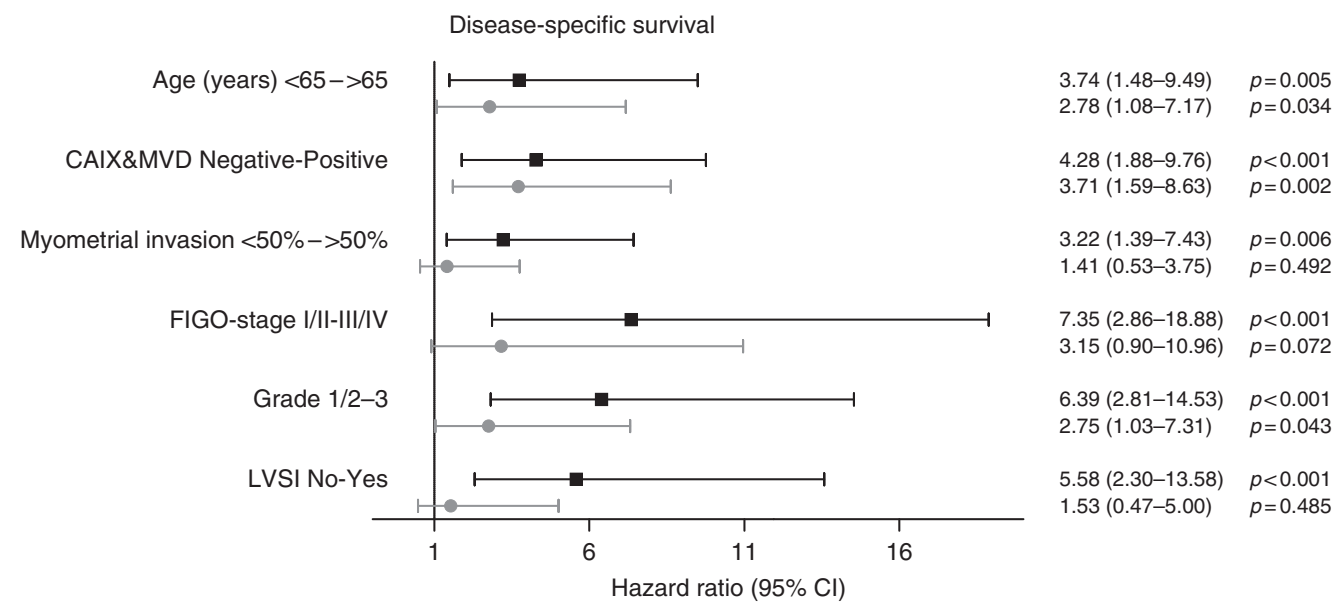

Fig. 3 Univariable and multivariable Cox regression analysis of clinicopathological parameters including carbonic anhydrase IX (CAIX) combined with vascular density for disease-specific survival (DSS). The hazard ratios with $95 \%$ confidence intervals are depicted by the black line. All risk factors significantly associated with DSS in univariable analysis were included in the multivariable Cox regression analysis, depicted by the grey lines 


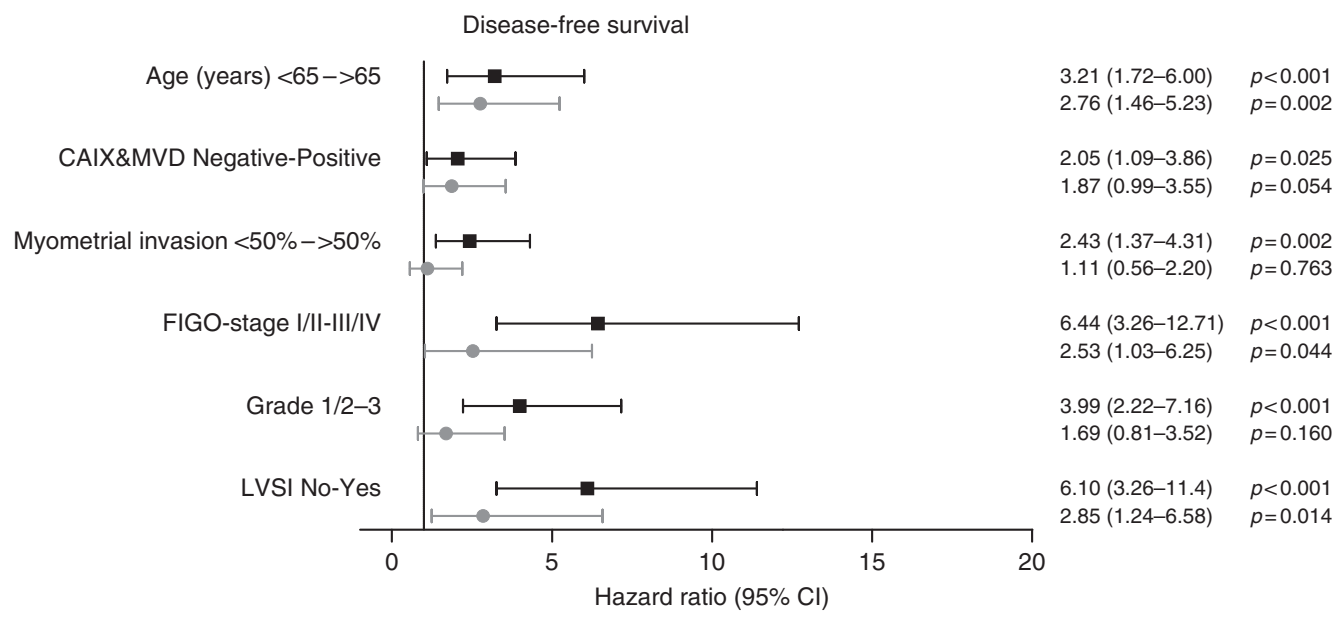

Fig. 4 Univariable and multivariable Cox regression analysis of clinicopathological parameters including carbonic anhydrase IX (CAIX) combined with vascular density for disease-free survival (DFS). All risk factors significantly associated with DFS in univariable analysis were included in the multivariable Cox regression analysis, depicted by the grey lines

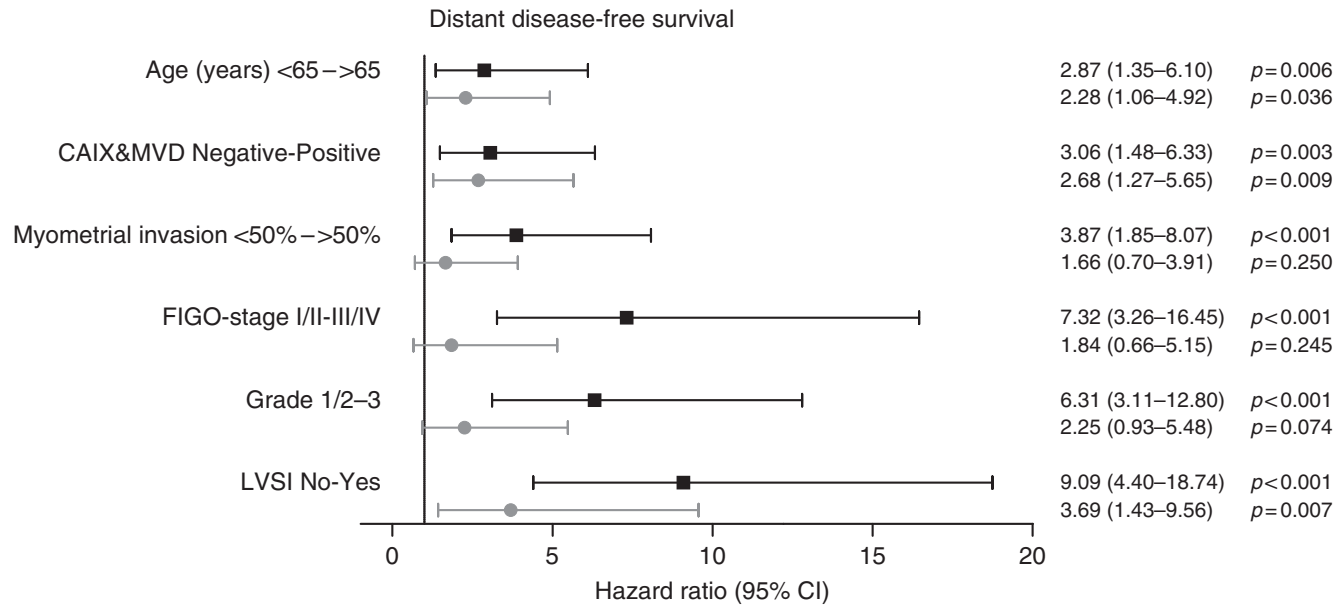

Fig. 5 Univariable and multivariable Cox regression analysis of clinicopathological parameters including carbonic anhydrase IX (CAIX) combined with vascular density for distant disease-free survival (DDFS). All risk factors significantly associated with DDFS in univariable analysis were included in the multivariable Cox regression analysis, depicted by the grey lines

several steps of this process, including promoting tumour cell survival by acquisition of a malignant phenotype and increased invasion in blood vessels. ${ }^{9}$

A recent meta-analysis has shown that high MVD was associated with several poor prognostic variables, including deep $\mathrm{MI}$, positive LVSI and poor outcome in EC, although heterogeneity due to differences in used antibodies and cut-off values hampers interpretation of these results. ${ }^{23}$ Biologically, intratumoural neoangiogenesis in response to hypoxia will promote the formation of vasculature with high degrees of permeability and potential for rapid growth. ${ }^{40}$ Our hypothesis that CAIX expression with high degrees of vascular density would be associated with unfavourable prognostic features and poor outcome was based both on the facilitation of haematogenous spread in areas with high angiogenesis and on the aggressive biological behaviour of tumour cells after hypoxia. ${ }^{41,42}$ HIF-1a is stabilised and accumulates under hypoxia, and activates transcription of numerous genes involved in angiogenesis, proliferation and $\mathrm{pH}$ regulation (VEGF, CAIX, glucose transporter-1). ${ }^{9}$ Our hypothesis was supported by the fact that ECs with positive CAIX expression and high vascular density had a decreased DSS compared to ECs with only one or none of both features. This observation supports the complex interplay underlying the metastatic processes. The observation that CAIX-positive ECs with high vascular density did not have more lymph node metastasis or local recurrences, but instead have more distant recurrences, could support the role of angiogenesis in the haematogenous rather than the lymphogenic metastatic process.

The obvious strengths of this study are the inclusion of a large and representative cohort of EC patients within the ENITEC network and the objective and reproducible measurement of CAIX and MVD using digital imaging analyses. However, there are some limitations that need to be addressed. Due to the retrospective nature of the study, there were missing values, specifically for LVSI and lymph node metastasis. Substantial LVSI is a stronger predictor for prognosis of EC compared to moderate LVSI. Also, LVSI is not routinely reported in the pathologic report at all centres. Therefore, we assumed that if substantial LVSI was present, it was reported, and if LVSI was not reported, no substantial LVSI was present. ${ }^{31}$ Missing cases were therefore coded as negative for LVSI. Separate analyses of patients with available LVSI status did not alter the results of the primary outcome (data not shown). Another general limitation in interpretation of CAIX and MVD is the lack of standardised criteria in the current literature, which hampers comparison of previous studies and this study. ${ }^{29,30}$ However, the applied digital 
techniques in this study enable objective and reproducible analyses without the need for extensive pathological expertise. With the integration of digital pathology into clinical practice, comparison of future studies with our results might be easier. ${ }^{43,44}$ Although widely used to quantify MVD, CD34 is known to also identify lymph vessels and stem cell populations, which theoretically could have led to an overestimation of our results. On the other hand, other antibodies, for example, CD31, also carry the risk of aspecific staining. Compared to CD31, CD34 staining is known to have stronger reactivity with endothelial cells, resulting in a lower risk of staining failure. ${ }^{45}$ Finally, generalisability to non-endometrioid subtypes can be questioned, as they comprise only $3.4 \%$ of the entire cohort. More research focused on this specific subgroup could help to strengthen these results.

This study identifies a group of patients with a poor DSS and DDFS based on CAIX and MVD. Given the increased risk of distant metastases, differential adjuvant treatment for these ECs could be explored either in the form of chemotherapy or, in the future, targeted therapies directed against angiogenesis. Because of the focal character of CAIX expression in the tumour tissue, performing the analysis on preoperative biopsies might be challenging, but visualisation of hypoxia and angiogenesis on FDG-PET/CT (fluorodeoxyglucose-positron emission tomography/computed tomography) scan and magnetic resonance imaging could be an alternative, as Berg et al. ${ }^{5}$ showed recently.

In summary, we have found that CAIX expression and high vascular density are prognostic markers for decreased survival in EC. Combining these two markers revealed that ECs with positive CAIX expression and high vascular density have an impaired outcome compared to ECs that have only one or none of both features. These patients experienced more distant recurrences, and therefore differential adjuvant treatment for these tumours should be explored.

\section{AUTHOR CONTRIBUTIONS}

All authors contributed to the manuscript. C.R., W.J.v.W., M.S.J.P.A., M.P.L.M.S., H.V.N. K.-V., L.F.A.G.M., J.B. and J.M.A.P. contributed to the study design. C.R., W.J.v.W., M.S.J. P.A., J.P.P., P.F.R., K.v.d.V., M.S., P.B., J. B., M.H., E.C., A.G.-M., A.R., G.M., C.K., J.T., I.S.H., J. H., M.K., V.W., L.M., E.J., M.P.L.M.S., S.v.d.B.-v.E., H.V.N.K.-V., X.M.-G., F.A., L.F.A.G.M., J.B., J.M.A.P. contributed to data collection. C.R., W.J.v.W., M.S.J.P.A., J.P.P., P.F.R, M.P.L.M.S., H.V.N.K.-V., L.F.A.G.M., J.B. and J.M.A.P. contributed to the data analysis. C.R., W.J.v.W., M.S.J.P.A., J.P.P., P.F.R, M.P.L.M.S., H.V.N.K.-V., L.F.A.G.M., J.B and J.M.A.P contributed to writing of the draft version of the manuscript. C.R., W.J.v.W., M.S.J.P.A., J.P.P., P.F.R., K.v. d.V., M.S., P.B., J.B., M.H., E.C., A.G.-M., A.R., G.M., C.K., J.T., I.S.H., J.H., M.K., V.W., L.M., E.J., M.P.L.M.S., S.v.d.B.-v.E., H.V.N.K.-V., X.M.-G., F.A., L.F.A.G.M., J.B. and J.M.A.P. have read, revised and have approved the final version of the manuscript being submitted for peer review.

\section{ADDITIONAL INFORMATION}

Supplementary information is available for this paper at https://doi.org/10.1038/ s41416-019-0461-2.

Competing interests: The authors declare no competing interests.

Ethical approval: This study was performed in accordance to the Declaration of Helsinki and was approved by the Institutional Review Board at the Radboud University Medical Centre.

Data availability: The datasets used during the current study can be made available from the corresponding author on reasonable request.

Note: This work is published under the standard license to publish agreement. After 12 months the work will become freely available and the license terms will switch to a Creative Commons Attribution 4.0 International (CC BY 4.0).
Publisher's note: Springer Nature remains neutral with regard to jurisdictional claims in published maps and institutional affiliations.

\section{REFERENCES}

1. Creasman, W. T., Morrow, C. P., Bundy, B. N., Homesley, H. D., Graham, J. E., Heller, P. B. Surgical pathologic spread patterns of endometrial cancer. A Gynecologic Oncology Group Study. Cancer 60(Suppl.), 2035-2041 (1987).

2. Bokhman, J. V. Two pathogenetic types of endometrial carcinoma. Gynecol. Oncol. 15, 10-17 (1983)

3. Nout, R. A., Smit, V. T., Putter, H., Jurgenliemk-Schulz, I. M., Jobsen, J. J., Lutgens, L. C. et al. Vaginal brachytherapy versus pelvic external beam radiotherapy for patients with endometrial cancer of high-intermediate risk (PORTEC-2): an openlabel, non-inferiority, randomised trial. Lancet 375, 816-823 (2010).

4. Trovik, J., Wik, E., Werner, H. M., Krakstad, C., Helland, H., Vandenput, I. et al. Hormone receptor loss in endometrial carcinoma curettage predicts lymph node metastasis and poor outcome in prospective multicentre trial. Eur. J. Cancer 49, 3431-3441 (2013).

5. Berg, A., Fasmer, K. E., Mauland, K. K., Ytre-Hauge, S., Hoivik, E. A., Husby, J. A. et al. Tissue and imaging biomarkers for hypoxia predict poor outcome in endometrial cancer. Oncotarget 7, 69844-69856 (2016).

6. Haldorsen, I. S., Stefansson, I., Gruner, R., Husby, J. A., Magnussen, I. J., Werner, H. $M$. et al. Increased microvascular proliferation is negatively correlated to tumour blood flow and is associated with unfavourable outcome in endometrial carcinomas. Br. J. Cancer 110, 107-114 (2014).

7. Gilkes, D. M., Semenza, G. L. \& Wirtz, D. Hypoxia and the extracellular matrix: drivers of tumour metastasis. Nat. Rev. Cancer 14, 430-439 (2014).

8. Bredholt, G., Mannelqvist, M., Stefansson, I. M., Birkeland, E., Bo, T. H., Oyan, A. M. et al. Tumor necrosis is an important hallmark of aggressive endometrial cancer and associates with hypoxia, angiogenesis and inflammation responses. Oncotarget 6, 39676-39691 (2015).

9. Span, P. N. \& Bussink, J. Biology of hypoxia. Semin. Nucl. Med 45, 101-109 (2015).

10. Rademakers, S. E., Span, P. N., Kaanders, J. H., Sweep, F. C., van der Kogel, A. J. \& Bussink, J. Molecular aspects of tumour hypoxia. Mol. Oncol. 2, 41-53 (2008).

11. Jiang, B. H., Semenza, G. L., Bauer, C. \& Marti, H. H. Hypoxia-inducible factor 1 levels vary exponentially over a physiologically relevant range of $\mathrm{O} 2$ tension. $\mathrm{Am}$. J. Physiol. 271(Part 1), C1172-C1180 (1996).

12. Semenza, G. L. HIF-1: mediator of physiological and pathophysiological responses to hypoxia. J. Appl. Physiol. (1985) 88, 1474-1480 (2000).

13. Ratcliffe, P. J., O'Rourke, J. F., Maxwell, P. H. \& Pugh, C. W. Oxygen sensing, hypoxia-inducible factor- 1 and the regulation of mammalian gene expression. J. Exp. Biol. 201 (Part 8), 1153-1162 (1998).

14. Ivanov, S. V., Kuzmin, I., Wei, M. H., Pack, S., Geil, L., Johnson, B. E. et al. Downregulation of transmembrane carbonic anhydrases in renal cell carcinoma cell lines by wild-type von Hippel-Lindau transgenes. Proc. Natl Acad. Sci. USA 95, 12596-12601 (1998).

15. Blancher, C. \& Harris, A. L. The molecular basis of the hypoxia response pathway: tumour hypoxia as a therapy target. Cancer Metastas. Rev. 17, 187-194 (1998).

16. Semenza, G. L. Defining the role of hypoxia-inducible factor 1 in cancer biology and therapeutics. Oncogene 29, 625-634 (2010).

17. Vleugel, M. M., Greijer, A. E., Shvarts, A., van der Groep, P., van Berkel, M., Aarbodem, Y. et al. Differential prognostic impact of hypoxia induced and diffuse HIF-1alpha expression in invasive breast cancer. J. Clin. Pathol. 58, 172-177 (2005).

18. Bussink, J., Kaanders, J. H. \& van der Kogel, A. J. Tumor hypoxia at the microregional level: clinical relevance and predictive value of exogenous and endogenous hypoxic cell markers. Radiother. Oncol. 67, 3-15 (2003).

19. Leek, R. D., Landers, R. J., Harris, A. L. \& Lewis, C. E. Necrosis correlates with high vascular density and focal macrophage infiltration in invasive carcinoma of the breast. Br. J. Cancer 79, 991-995 (1999).

20. Kato, T., Kimura, T., Miyakawa, R., Tanaka, S., Fujii, A., Yamamoto, K. et al. Clinicopathologic study of angiogenesis in Japanese patients with breast cancer. World J. Surg. 21, 49-56 (1997).

21. Yu, S., Sun, J., Zhang, J., Xu, X., Li, H., Shan, B. et al. Aberrant expression and association of VEGF and DII4/Notch pathway molecules under hypoxia in patients with lung cancer. Histol. Histopathol. 28, 277-284 (2013).

22. Pijnenborg, J. M., Wijnakker, M., Hagelstein, J., Delvoux, B., Groothuis, P. G. Hypoxia contributes to development of recurrent endometrial carcinoma. Int. J. Gynecol. Cancer 17, 897-904 (2007).

23. Wang, J. Z., Xiong, Y. J., Man, G. C. W., Chen, X. Y., Kwong, J., Wang, C. C. Clinicopathological and prognostic significance of blood microvessel density in endometrial cancer: a meta-analysis and subgroup analysis. Arch. Gynecol. Obstet. 297, 731-740 (2018). 
24. van der Putten, L. J., Visser, N. C., van de Vijver, K., Santacana, M., Bronsert, P., Bulten, J. et al. L1CAM expression in endometrial carcinomas: an ENITEC collaboration study. Br. J. Cancer 115, 716-724 (2016).

25. van der Putten, L. J. M., Visser, N. C. M., van de Vijver, K., Santacana, M., Bronsert, P., Bulten, J. et al. Added value of estrogen receptor, progesterone receptor, and L1 cell adhesion molecule expression to histology-based endometrial carcinoma recurrence prediction models: an ENITEC Collaboration Study. Int. J. Gynecol. Cancer. 28, 514-523 (2018).

26. Rijken, P. F., Bernsen, H. J. \& van der Kogel, A. J. Application of an image analysis system to the quantitation of tumor perfusion and vascularity in human glioma xenografts. Micro. Res. 50, 141-153 (1995).

27. Stegeman, H., Rademakers, S. E., Span, P. N., Takes, R. P., van der Kogel, A. J., Kaanders, J. H. et al. Hypoxia, metabolism, and growth factor signaling in head and neck squamous cell carcinoma: correlation between primary and xenograft tumors. Head. Neck 36, 1288-1295 (2014).

28. Weidner, N., Semple, J. P., Welch, W. R. \& Folkman, J. Tumor angiogenesis and metastasis-correlation in invasive breast carcinoma. N. Engl. J. Med. 324, 1-8 (1991).

29. Seeber, L. M., Horree, N., van der Groep, P., van der Wall, E., Verheijen, R. H. \& van Diest, P. J. Necrosis related HIF-1alpha expression predicts prognosis in patients with endometrioid endometrial carcinoma. BMC Cancer 10, 307 (2010).

30. Sadlecki, P., Bodnar, M., Grabiec, M., Marszalek, A., Walentowicz, P., Sokup, A. et al. The role of hypoxia-inducible factor-1 alpha, glucose transporter-1, (GLUT-1) and carbon anhydrase IX in endometrial cancer patients. Biomed. Res. Int. 2014, 616850 (2014).

31. Bosse, T., Peters, E. E., Creutzberg, C. L., Jurgenliemk-Schulz, I. M., Jobsen, J. J., Mens, J. W. et al. Substantial lymph-vascular space invasion (LVSI) is a significant risk factor for recurrence in endometrial cancer-a pooled analysis of PORTEC 1 and 2 trials. Eur. J. Cancer (Oxford, England: 1990) 51, 1742-1750 (2015).

32. Koskas, M., Bassot, K., Graesslin, O., Aristizabal, P., Barranger, E., Clavel-Chapelon, F. et al. Impact of lymphovascular space invasion on a nomogram for predicting lymph node metastasis in endometrial cancer. Gynecol. Oncol. 129, 292-297 (2013).

33. Span, P. N., Bussink, J., Manders, P., Beex, L. V. \& Sweep, C. G. Carbonic anhydrase9 expression levels and prognosis in human breast cancer: association with treatment outcome. Br. J. Cancer 89, 271-276 (2003).
34. Hussain, S. A., Ganesan, R., Reynolds, G., Gross, L., Stevens, A., Pastorek, J. et al. Hypoxia-regulated carbonic anhydrase IX expression is associated with poor survival in patients with invasive breast cancer. Br. J. Cancer 96, 104-109 (2007).

35. Cetin, B., Gonul, I. I., Gumusay, O., Bilgetekin, I., Algin, E., Ozet, A. et al. Carbonic anhydrase IX is a prognostic biomarker in glioblastoma multiforme. Neuropathology. https://doi.org/10.1111/neup.12485 (2018).

36. Finkelmeier, F., Canli, O., Peiffer, K. H., Walter, D., Tal, A., Koch, C. et al. Circulating hypoxia marker carbonic anhydrase IX (CA9) in patients with hepatocellular carcinoma and patients with cirrhosis. PLOS ONE 13, e0200855 (2018).

37. Kirkpatrick, J. P., Rabbani, Z. N., Bentley, R. C., Hardee, M. E., Karol, S., Meyer, J. et al. Elevated CAIX expression is associated with an increased risk of distant failure in early-stage cervical cancer. Biomark Insights 3, 45-55 (2008).

38. Chia, S. K., Wykoff, C. C., Watson, P. H., Han, C., Leek, R. D., Pastorek, J. et al. Prognostic significance of a novel hypoxia-regulated marker, carbonic anhydrase IX, in invasive breast carcinoma. J. Clin. Oncol. 19, 3660-3668 (2001).

39. Chang, J. \& Erler, J. Hypoxia-mediated metastasis. Adv. Exp. Med. Biol. 772, 55-81 (2014).

40. Baluk, P., Morikawa, S., Haskell, A., Mancuso, M. \& McDonald, D. M. Abnormalities of basement membrane on blood vessels and endothelial sprouts in tumors. Am. J. Pathol. 163, 1801-1815 (2003).

41. Yeo, C. D., Kang, N., Choi, S. Y., Kim, B. N., Park, C. K., Kim, J. W. et al. The role of hypoxia on the acquisition of epithelial-mesenchymal transition and cancer stemness: a possible link to epigenetic regulation. Korean J. Intern. Med. 32, 589-599 (2017)

42. Bao, B., Azmi, A. S., Ali, S., Ahmad, A., Li, Y., Banerjee, S. et al. The biological kinship of hypoxia with CSC and EMT and their relationship with deregulated expression of miRNAs and tumor aggressiveness. Biochim. Biophys. Acta 1826, 272-296 (2012).

43. Lloyd, M. C., Monaco, J. P. \& Bui, M. M. Image analysis in surgical pathology. Surg. Pathol. Clin. 9, 329-337 (2016).

44. Bueno, G., Fernandez-Carrobles, M. M., Deniz, O. \& Garcia-Rojo, M. New trends of emerging technologies in digital pathology. Pathobiology 83, 61-69 (2016).

45. Uzzan, B., Nicolas, P., Cucherat, M. \& Perret, G. Y. Microvessel density as a prognostic factor in women with breast cancer: a systematic review of the literature and meta-analysis. Cancer Res 64, 2941-2955 (2004).. 